\title{
Correction to: Adiponectin aggravates bone erosion by promoting osteopontin production in synovial tissue of rheumatoid arthritis
}

Jie Qian ${ }^{1,2}$, Lingxiao Xu ${ }^{1 *}$, Xiaoxuan Sun ${ }^{1}$, Yani Wang ${ }^{1}$, Wenhua Xuan ${ }^{1}$, Qian Zhang ${ }^{1}$, Pengfei Zhao ${ }^{1}$, Qin Wu ${ }^{1}$, Rui Liu', Nan Che', Fang Wang ${ }^{3}$, Wenfeng $\operatorname{Tan}^{1}$ and Miaojia Zhang ${ }^{1 *}$

\section{Correction}

Unfortunately, after publication of this article [1], it was noticed that the panel for Fig. 4b was inadvertently obscured during the production process. The full, correct Fig. 4 can be seen below and the original article has been corrected to reflect this.

\section{Author details}

'Department of Rheumatology, The First Affiliated Hospital of Nanjing Medical University, 300 Guangzhou Road, Nanjing 210029, China.

${ }^{2}$ Department of Rheumatology, Affiliated Hospital of Nantong University, 20 Xisi Road, Nantong 226001, China. ${ }^{3}$ Department of Cardiology, The First Affiliated Hospital of Nanjing Medical University, 300 Guangzhou Road, Nanjing 210029, China.

Received: 23 May 2018 Accepted: 23 May 2018

Published online: 13 June 2018

\section{Reference}

1. Qian J, Xu L, Sun X, Wang Y, Xuan W, Zhang Q, Zhao P, Wu Q, Liu R, Che N, Wang F, Tan W, Zhang M. Adiponectin aggravates bone erosion by promoting osteopontin production in synovial tissue of rheumatoid arthritis. Arthritis Research \& Therapy. 2018;20:26. https://doi.org/10.1186/s13075-018-1526-y.

\footnotetext{
* Correspondence: mjzhang@njmu.edu.cn; lingxiao32@163.com 'Department of Rheumatology, The First Affiliated Hospital of Nanjing Medical University, 300 Guangzhou Road, Nanjing 210029, China
} 


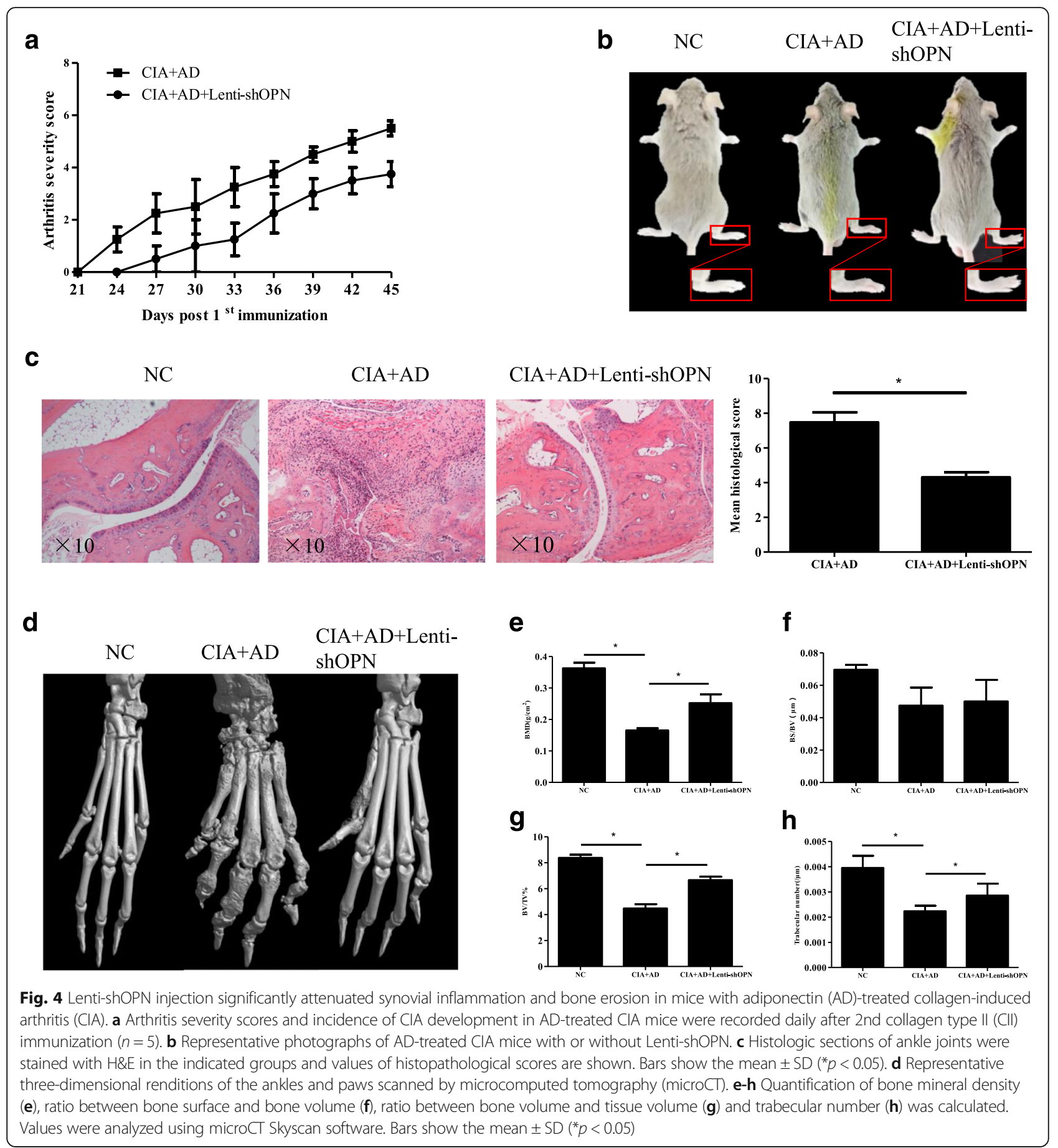

